\title{
The genome sequence of the European hornet, Vespa crabro
}

\section{Linnaeus, 1758 [version 1; peer review: 1 approved]}

Liam M. Crowley (iD),

University of Oxford and Wytham Woods Genome Acquisition Lab,

Darwin Tree of Life Barcoding collective,

Wellcome Sanger Institute Tree of Life programme,

Wellcome Sanger Institute Scientific Operations: DNA Pipelines collective, Tree of Life Core Informatics collective, Darwin Tree of Life Consortium

${ }^{1}$ Department of Zoology, University of Oxford, Oxford, UK

V1 First published: 27 Jan 2022, 7:27

https://doi.org/10.12688/wellcomeopenres.17546.1

Latest published: 27 Jan 2022, 7:27

https://doi.org/10.12688/wellcomeopenres.17546.1

\section{Abstract}

We present a genome assembly from an individual female Vespa crabro (the European hornet; Arthropoda; Insecta; Hymenoptera; Vespidae). The genome sequence is 230 megabases in span. The majority of the assembly (94.93\%) is scaffolded into 25 chromosomal pseudomolecules.

Keywords

Vespa crabro, European hornet, genome sequence, chromosomal, Hymenoptera

This article is included in the Tree of Life gateway.

\section{Open Peer Review \\ Approval Status}

1

version 1

27 Jan 2022

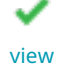

1. Artem Nedoluzhko ID, Russian Academy of Sciences, Saint Petersburg, Russian

Federation

Any reports and responses or comments on the article can be found at the end of the article. 
Corresponding author: Darwin Tree of Life Consortium (mark.blaxter@sanger.ac.uk)

Author roles: Crowley LM: Investigation, Resources, Writing - Original Draft Preparation;

Competing interests: No competing interests were disclosed.

Grant information: This work was supported by Wellcome through core funding to the Wellcome Sanger Institute (206194) and the Darwin Tree of Life Discretionary Award (218328).

The funders had no role in study design, data collection and analysis, decision to publish, or preparation of the manuscript.

Copyright: (c) 2022 Crowley LM et al. This is an open access article distributed under the terms of the Creative Commons Attribution License, which permits unrestricted use, distribution, and reproduction in any medium, provided the original work is properly cited.

How to cite this article: Crowley LM, University of Oxford and Wytham Woods Genome Acquisition Lab, Darwin Tree of Life Barcoding collective et al. The genome sequence of the European hornet, Vespa crabro Linnaeus, 1758 [version 1; peer review: 1 approved] Wellcome Open Research 2022, 7:27 https://doi.org/10.12688/wellcomeopenres.17546.1

First published: 27 Jan 2022, 7:27 https://doi.org/10.12688/wellcomeopenres.17546.1 


\section{Species taxonomy}

Eukaryota; Metazoa; Ecdysozoa; Arthropoda; Hexapoda; Insecta; Pterygota; Neoptera; Endopterygota; Hymenoptera; Apocrita; Aculeata; Vespoidea; Vespidae; Vespinae; Vespa; Vespa crabro Linnaeus, 1758 (NCBI:txid7445).

\section{Background}

The European hornet, Vespa crabro, is the largest social wasp in Europe, with workers measuring up to $23 \mathrm{~mm}$ and queens up to $30 \mathrm{~mm}$ (Archer \& Turner, 2014). It is common and widespread throughout much of the palearctic, including throughout England and Wales, and has been introduced into North America (Archer, 1992). Vespa crabro occurs in a wide range of habitats but is particularly associated with mature deciduous woodlands and urban environments. The head and majority of the metasoma are predominantly yellow and the mesosoma, anterior segments of the metasoma and legs are reddish-brown with varying extents of black markings.

This species is eusocial, living in colonies with a queen, workers and males. Whilst workers are capable of producing male offspring, this is rare due to the action of worker policing (Foster et al., 2002). Colonies are founded by overwintered queens from early April, with the first workers appearing in late June to early July (Bunn, 1988b). Nests are constructed out of a paper-like substance produced from the pulp of decaying wood mixed with saliva, often incorporating bark and twig fragments (Spredbery, 1973). The nest consists of up to around 1400 hexagonal cells arranged into 3-8 combs, covered by a nest envelope (Archer, 1993; Archer, 2008). Nests are usually located in aerial situations, particularly in hollow trees, but are also commonly located in human structures such as attics and outbuildings. Colonies may relocate the nest should it exceed the available space (Pawlyszyn, 1992). Nests are occasionally located underground, but such nests are more frequently relocated (Archer \& Turner, 2014). The nests are well characterised as containing many species of inquilines, predators and parasitoids. Colonies build up throughout the summer, before the reproductive males and gynes are produced in September. Most queens are singularly mated but double and triple mating also occurs, although paternity of the offspring produced by multiply-mated queens is heavily biased to a single male (Foster et al., 1999). Workers may persist until October or occasionally early November (Archer, 1993; Bunn, 1988b).

Female hornets are generalist predators, catching, killing, and preparing prey of various arthropods to take back to the nest to feed to the developing brood. Prey includes other species of social wasp, honeybees, flies, butterflies, moths and spiders (Pawlyszyn, 1994). In particular, returning honeybee foragers are frequently taken, although this species does not inflict a considerable impact on honeybee colonies due to honeybee defensive behaviours (Baracchi et al., 2010). Vespa crabro also often frequents sap runs, where it feeds on the exudations, and is also known to rig bark twigs to stimulate sap flow (Bunn, 1988a; Edwards, 1980). Ivy (Hedera helix) flowers are visited when in bloom to feed on nectar (Pawlyszyn, 1994), particularly by males. The trophic preferences of this species overlap with the invasive Vespa velutina, meaning that where these species co-occur, V. crabro may be outperformed in instances of interspecific competition (Cini et al., 2018).

The species is not particularly aggressive, although females may sting if provoked or in defence of the nest. Major venom components, such as prepromastoparan, vespid chemotactic peptide precursor and vespakinin, are more highly enriched that in some other species of Vespa, meaning that the venom likely has a greater toxicity (Yoon et al., 2015).

\section{Genome sequence report}

The genome was sequenced from a single female $V$. crabro (Figure 1) collected from Wytham Woods, Oxfordshire, UK

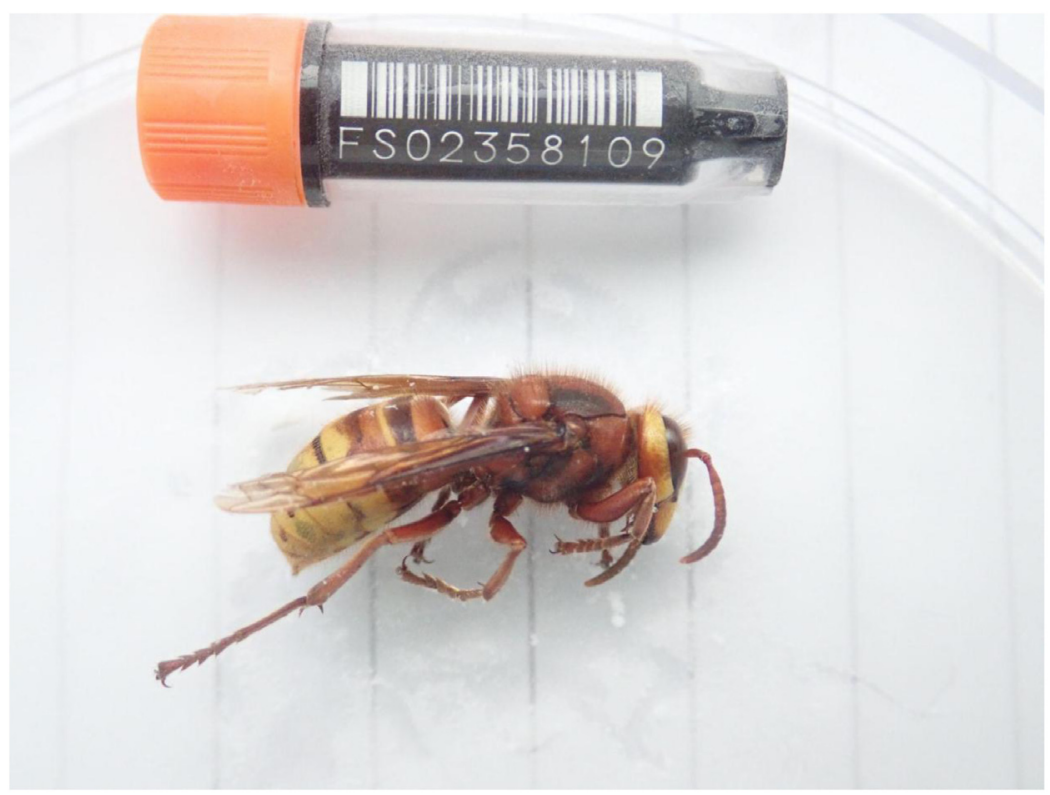

Figure 1. Image of the iyVesCrab1 specimen taken during preservation and processing. 
(latitude 51.77, longitude -1.338). A total of 70-fold coverage in Pacific Biosciences single-molecule long reads and 104fold coverage in 10X Genomics read clouds were generated. Primary assembly contigs were scaffolded with chromosome conformation Hi-C data. Manual assembly curation corrected $96 \mathrm{missing} / \mathrm{misjoins}$, reducing the scaffold number by $41.67 \%$, and increasing the scaffold N50 by $113.35 \%$ and assembly length by $0.01 \%$.

The final assembly has a total length of $230 \mathrm{Mb}$ in 106 sequence scaffolds with a scaffold N50 of $10 \mathrm{Mb}$ (Table 1). Of the assembly sequence, $94.9 \%$ was assigned to 25 chromosomal-level scaffolds (numbered by sequence length) (Figure 2-Figure 5; Table 2). The assembly has a BUSCO v5.1.2 (Simão et al., 2015) completeness of 96.5\% (single $96.3 \%$, duplicated $0.3 \%$ ) using the hymenoptera_odb10 reference set. While not fully phased, the assembly deposited is of one haplotype. Contigs corresponding to the second haplotype have also been deposited.
The assembly was constructed into 25 chromosomes and seems in agreement with the expected karyotype from Hoshiba et al. (1989). Large centromeric regions were observed in the Hi-C map, notably on SUPER_1, SUPER_12 and SUPER_16 ( 2-3 Mbp in size), and could explain the skew in genome content to AT. Scaffold_5 ( 6 Mbp in size) appears to be a collapsed centromeric repeat and could not be confidently placed.

\section{Methods}

Sample acquisition and DNA extraction

A single female $V$. crabro was collected from Wytham Woods, Oxfordshire, UK (latitude 51.774, longitude -1.332) by Liam Crowley, University of Oxford, using a net. The sample was identified by the same individual and snap-frozen on dry ice.

DNA was extracted at the Tree of Life laboratory, Wellcome Sanger Institute. The iyVesCrab1 sample was weighed and dissected on dry ice with tissue set aside for $\mathrm{Hi}-\mathrm{C}$ sequencing.

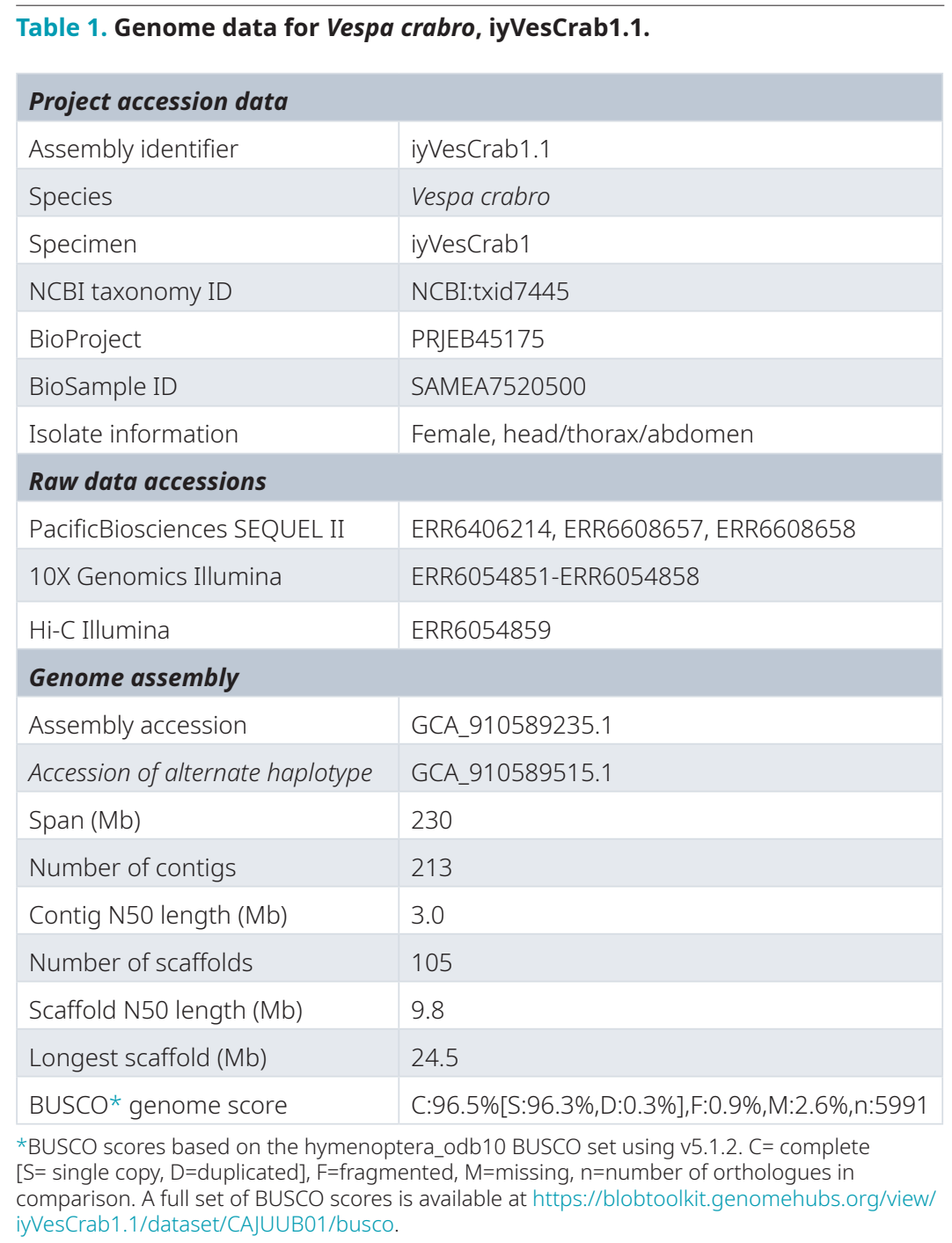




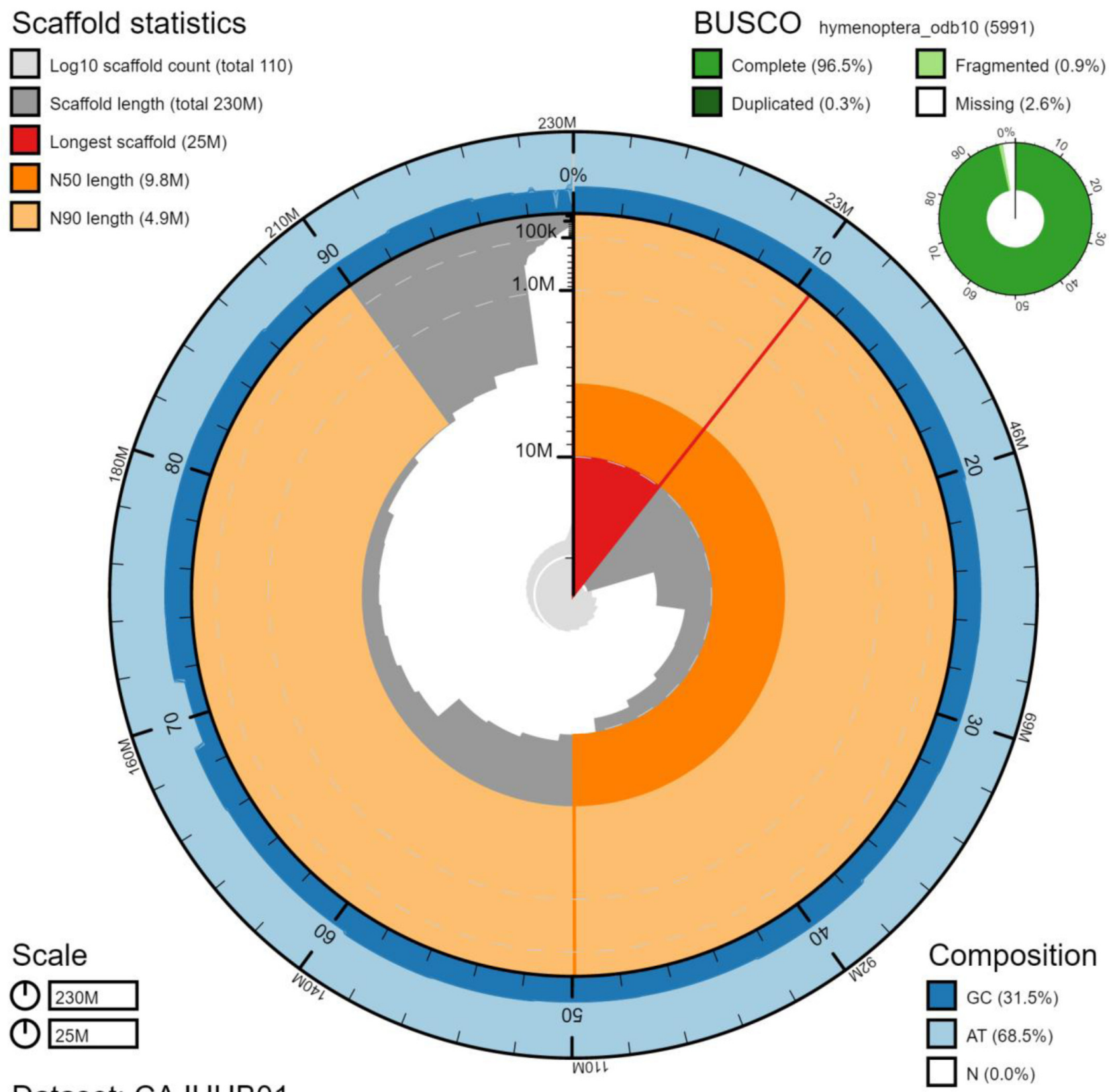

\section{Dataset: CAJUUB01}

Figure 2. Genome assembly of Vespa crabro, iyVesCrab1: metrics. The BlobToolKit Snailplot shows N50 metrics and BUSCO gene completeness. The main plot is divided into 1,000 size-ordered bins around the circumference with each bin representing $0.1 \%$ of the $229,601,916$ bp assembly. The distribution of scaffold lengths is shown in dark grey with the plot radius scaled to the longest scaffold present in the assembly $(24,517,513 \mathrm{bp}$, shown in red). Orange and pale-orange arcs show the N50 and N90 chromosome lengths $(9,767,562$ and 4,871,713 bp), respectively. The pale grey spiral shows the cumulative chromosome count on a log scale with white scale lines showing successive orders of magnitude. The blue and pale-blue area around the outside of the plot shows the distribution of GC, AT and $\mathrm{N}$ percentages in the same bins as the inner plot. A summary of complete, fragmented, duplicated and missing BUSCO genes in the hymenoptera_odb10 set is shown in the top right. An interactive version of this figure is available at https://blobtoolkit.genomehubs. org/view/iyvesCrab1.1/dataset/CAJUUB01/snail.

Thorax tissue was disrupted using a Nippi Powermasher fitted with a BioMasher pestle. Fragment size analysis of 0.01-0.5 ng of DNA was then performed using an Agilent FemtoPulse.
High molecular weight (HMW) DNA was extracted using the Qiagen MagAttract HMW DNA extraction kit. Low molecular weight DNA was removed from a 200-ng aliquot of extracted 


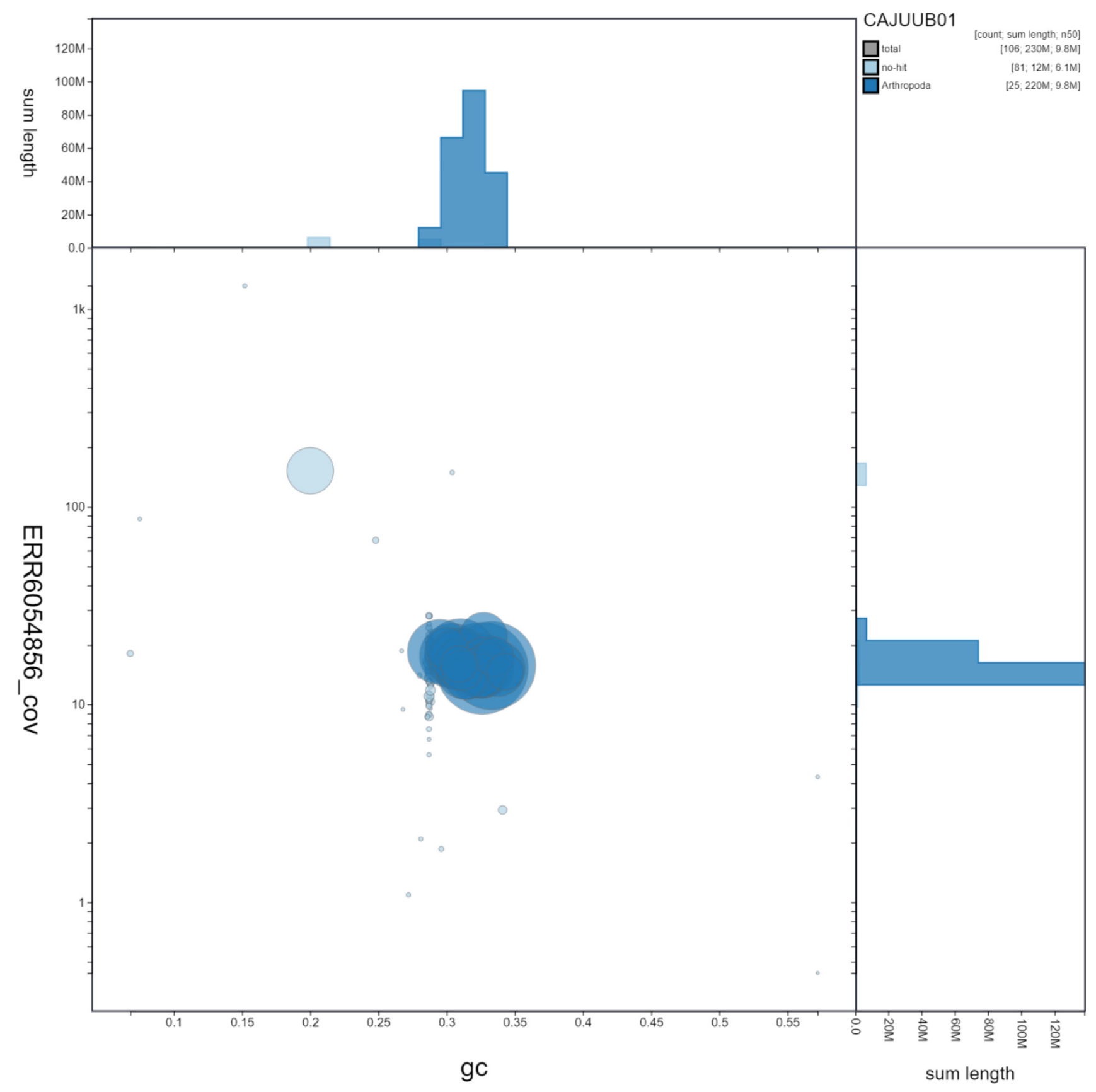

Figure 3. Genome assembly of Vespa crabro, iyVesCrab1: GC coverage. BlobToolKit GC-coverage plot. Scaffolds are coloured by phylum. Circles are sized in proportion to scaffold length. Histograms show the distribution of scaffold length sum along each axis. An interactive version of this figure is available at https://blobtoolkit.genomehubs.org/view/iyVesCrab1.1/dataset/CAJUUB01/blob.

DNA using $0.8 \mathrm{X}$ AMpure $\mathrm{XP}$ purification kit prior to $10 \mathrm{X}$ Chromium sequencing; a minimum of $50 \mathrm{ng}$ DNA was submitted for $10 \mathrm{X}$ sequencing. HMW DNA was sheared into an average fragment size between $12-20 \mathrm{~kb}$ in a Megaruptor 3 system with speed setting 30. Sheared DNA was purified by solid-phase reversible immobilisation using AMPure PB beads with a $1.8 \mathrm{X}$ ratio of beads to sample to remove the shorter fragments and concentrate the DNA sample. The concentration of the sheared and purified DNA was assessed using a Nanodrop spectrophotometer and Qubit Fluorometer and Qubit dsDNA High Sensitivity Assay kit. Fragment size distribution was evaluated by running the sample on the FemtoPulse system.

\section{Sequencing}

Pacific Biosciences HiFi circular consensus and 10X Genomics read cloud sequencing libraries were constructed according 


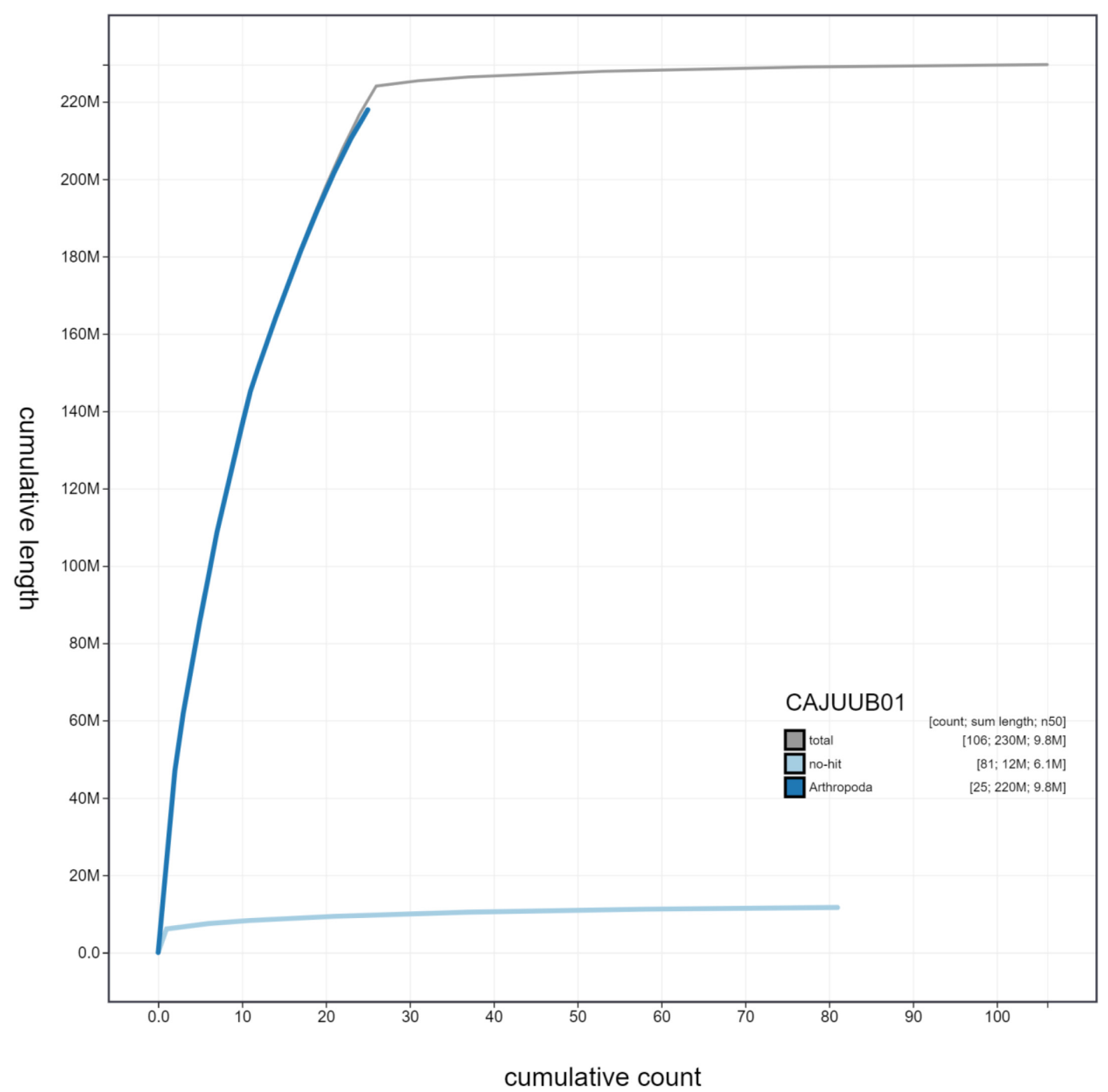

Figure 4. Genome assembly of Vespa crabro, iyVesCrab1: cumulative sequence. BlobToolKit cumulative sequence plot. The grey line shows cumulative length for all scaffolds. Coloured lines show cumulative lengths of scaffolds assigned to each phylum using the buscogenes taxrule. An interactive version of this figure is available at https://blobtoolkit.genomehubs.org/view/iyvesCrab1.1/dataset/ CAJUUB01/cumulative.

to the manufacturers' instructions. Sequencing was performed by the Scientific Operations core at the Wellcome Sanger Institute on Pacific Biosciences SEQUEL II and Illumina HiSeq X instruments. Hi-C data were generated from remaining thorax tissue using the Arima v2.0 kit and sequenced on an Illumina NovaSeq 6000 instrument.

\section{Genome assembly}

Assembly was carried out with Hifiasm (Cheng et al., 2021). Haplotypic duplication was identified and removed with purge_dups (Guan et al., 2020). Scaffolding with Hi-C data (Rao et al., 2014) was carried out with SALSA2 (Ghurye et al., 2019). The Hi-C scaffolded assembly was polished with 


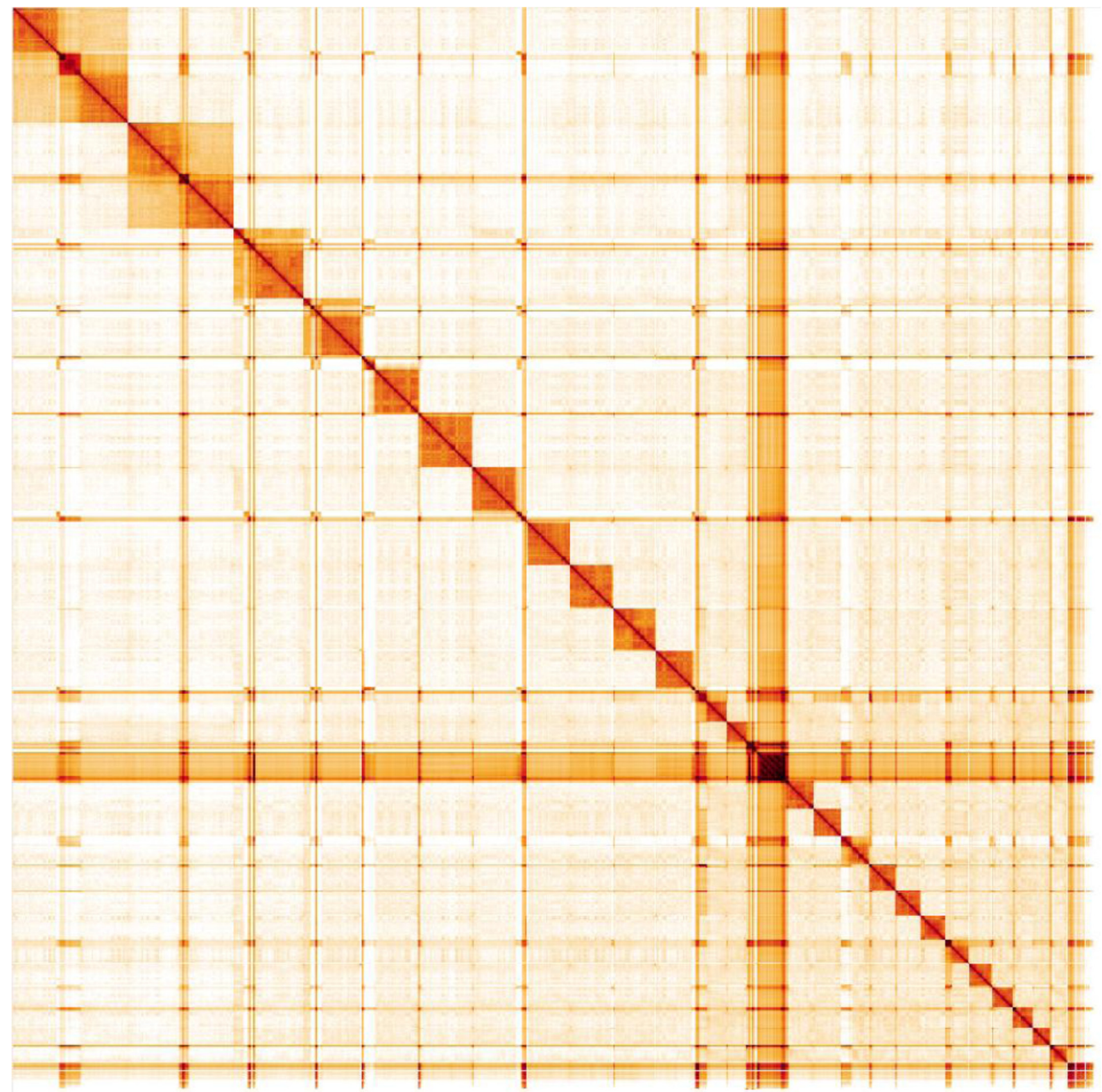

Figure 5. Genome assembly of Vespa crabro, iyVesCrab1: Hi-C contact map. Hi-C contact map of the iyVesCrab1.1 assembly, visualised in HiGlass. Chromosomes are shown in size order from left to right and top to bottom.

the 10X Genomics Illumina data by aligning to the assembly with longranger align, calling variants with freebayes (Garrison \& Marth, 2012). One round of the Illumina polishing was applied. The mitochondrial genome was assembled with MitoHiFi (Uliano-Silva et al., 2021), which performed annotation using MitoFinder (Allio et al., 2020). The assembly was checked for contamination and corrected using the gEVAL system (Chow et al., 2016) as described previously (Howe et al., 2021). Manual curation (Howe et al., 2021) was performed using gEVAL, HiGlass (Kerpedjiev et al., 2018) and Pretext. The genome was analysed within the BlobToolKit environment
(Challis et al., 2020). Table 3 contains a list of all software tool versions used, where appropriate.

\section{Ethics/compliance issues}

The materials that have contributed to this genome note have been supplied by a Darwin Tree of Life Partner. The submission of materials by a Darwin Tree of Life Partner is subject to the Darwin Tree of Life Project Sampling Code of Practice. By agreeing with and signing up to the Sampling Code of Practice, the Darwin Tree of Life Partner agrees they will meet the legal and ethical requirements and standards set out within 
Table 2. Chromosomal pseudomolecules in the genome assembly of Vespa crabro, iyVesCrab1.1.

\begin{tabular}{|c|c|c|c|}
\hline INSDC accession & Chromosome & Size (Mb) & GC\% \\
\hline OU342400.1 & 1 & 24.52 & 32.6 \\
\hline OU342401.1 & 2 & 22.54 & 33.3 \\
\hline OU342402.1 & 3 & 14.88 & 31.0 \\
\hline OU342403.1 & 4 & 12.12 & 30.4 \\
\hline OU342404.1 & 5 & 12.04 & 29.5 \\
\hline OU342405.1 & 6 & 11.60 & 32.6 \\
\hline OU342406.1 & 7 & 10.99 & 30.9 \\
\hline OU342407.1 & 8 & 9.77 & 31.4 \\
\hline OU342408.1 & 9 & 9.21 & 32.5 \\
\hline OU342409.1 & 10 & 8.84 & 33.7 \\
\hline OU342410.1 & 11 & 8.62 & 30.8 \\
\hline OU342411.1 & 12 & 6.64 & 30.9 \\
\hline OU342412.1 & 13 & 6.37 & 32.7 \\
\hline OU342413.1 & 14 & 5.95 & 32.1 \\
\hline OU342414.1 & 15 & 5.84 & 32.6 \\
\hline OU342415.1 & 16 & 5.79 & 32.7 \\
\hline OU342416.1 & 17 & 5.72 & 32.9 \\
\hline OU342417.1 & 18 & 5.29 & 32.1 \\
\hline OU342418.1 & 19 & 5.27 & 32.5 \\
\hline OU342419.1 & 20 & 4.92 & 30.0 \\
\hline OU342420.1 & 21 & 4.87 & 31.4 \\
\hline OU342421.1 & 22 & 4.45 & 31.1 \\
\hline OU342422.1 & 23 & 4.32 & 33.5 \\
\hline OU342423.1 & 24 & 3.72 & 34.3 \\
\hline OU342424.1 & 25 & 3.65 & 30.9 \\
\hline OU342425.1 & MT & 0.02 & 15.2 \\
\hline- & Unplaced & 11.64 & 24.1 \\
\hline
\end{tabular}

Table 3. Software tools used.

\begin{tabular}{|l|l|l|}
\hline Software tool & Version & Source \\
\hline Hifiasm & 0.12 & Cheng et al., 2021 \\
\hline purge_dups & 1.2 .3 & Guan et al., 2020 \\
\hline SALSA2 & 2.2 & Ghurye et al., 2019 \\
\hline longranger align & 2.2 .2 & $\begin{array}{l}\text { https://support.10xgenomics.com/ } \\
\text { genome-exome/software/pipelines/latest/ } \\
\text { advanced/other-pipelines }\end{array}$ \\
\hline
\end{tabular}




\begin{tabular}{|l|l|l|}
\hline Software tool & Version & Source \\
\hline freebayes & $\begin{array}{l}\text { v1.3.1-17- } \\
\text { gaa2ace8 }\end{array}$ & Garrison \& Marth, 2012 \\
\hline MitoHiFi & 1.0 & Uliano-Silva et al. (2021) \\
\hline gEVAL & N/A & Chow et al., 2016 \\
\hline HiGlass & 1.11 .6 & Kerpedjiev et al., 2018 \\
\hline PretextView & 0.0 .4 & https://github.com/wtsi-hpag/PretextView \\
\hline BlobToolKit & 2.6 .4 & Challis et al., 2020 \\
\hline
\end{tabular}

this document in respect of all samples acquired for, and supplied to, the Darwin Tree of Life Project. Each transfer of samples is further undertaken according to a Research Collaboration Agreement or Material Transfer Agreement entered into by the Darwin Tree of Life Partner, Genome Research Limited (operating as the Wellcome Sanger Institute), and in some circumstances other Darwin Tree of Life collaborators.

\section{Data availability}

European Nucleotide Archive: Vespa crabro (European hornet). Accession number PRJEB45175: https://identifiers.org/ena.embl: PRJEB45175

The genome sequence is released openly for reuse. The $\mathrm{V}$. crabro genome sequencing initiative is part of the Darwin Tree of Life (DToL) project. All raw sequence data and the assembly have been deposited in INSDC databases. The genome will be annotated and presented through the Ensembl pipeline at the European Bioinformatics Institute. Raw data and assembly accession identifiers are reported in Table 1.

\section{Author information}

Members of the University of Oxford and Wytham Woods Genome Acquisition Lab are listed here: https://doi.org/10.5281/ zenodo.5746938.

Members of the Darwin Tree of Life Barcoding collective are listed here: https://doi.org/10.5281/zenodo.5744972.

Members of the Wellcome Sanger Institute Tree of Life programme are listed here: https://doi.org/10.5281/zenodo. 5744840 .

Members of Wellcome Sanger Institute Scientific Operations: DNA Pipelines collective are listed here: https://doi.org/10.5281/ zenodo.5746904.

Members of the Tree of Life Core Informatics collective are listed here: https://doi.org/10.5281/zenodo.5743293.

Members of the Darwin Tree of Life Consortium are listed here: https://doi.org/10.5281/zenodo.5638618.
Allio R, Schomaker-Bastos A, Romiguier J, et al.: MitoFinder: Efficient automated large-scale extraction of mitogenomic data in target enrichment phylogenomics. Mol Ecol Resour. 2020; 20(4): 892-905. PubMed Abstract | Publisher Full Text | Free Full Text

Archer ME: The Taxonomy of Vespa Crabro L. and V. Dybowskii Andre (Hym., Vespinae). Entomologist's Monthly Magazine. 1992; 128: 157-63.

Reference Source

Archer ME: The Life History and Colonial Characteristics of the Hornet Vespa Crabro L.(Hym., Vespinae). Entomologist's Monthly Magazine. 1993; 129(1548-1551): 151-63.

Reference Source

Archer ME: Taxonomy, Distribution and Nesting Biology of Species of the Genera Provespa Ashmead and Vespa Linnaeus (Hymenoptera, Vespidae). Entomologist's Monthly Magazine. 2008; 144(1727): 69.

Archer ME, Turner JA: The Vespoid Wasps:(Tiphiidae, Mutillidae, Sapygidae, Scoliidae and Vespidae) of the British Isles. In Royal Entomological Society. 2014.

Reference Source

Baracchi D, Cusseau G, Pradella D, et al.: Defence reactions of Apis mellifera ligustica against attacks from the European hornet Vespa crabro. Ethol Ecol
Evol. 2010; 22(3): 281-94.

Publisher Full Text

Bunn DS: Observations on the Foraging Habits of the Hornet Vespa Crabro L.(Hym., Vespidae). Entomologist's Monthly Magazine. 1988a; 124(1492-1495): 187-93.

Reference Source

Bunn DS: The Nesting Cycle of the Hornet Vespa Crabro L.(Hym., Vespidae). Entomologist's Monthly Magazine. 1988b; 124(1488-1491): 117-22.

Reference Source

Challis R, Richards E, Rajan J, et al: BlobToolKit - Interactive Quality Assessment of Genome Assemblies. G3 (Bethesda). 2020; 10(4): 1361-74. PubMed Abstract | Publisher Full Text | Free Full Text

Cheng $\mathrm{H}$, Concepcion GT, Feng $\mathrm{X}$, et al:: Haplotype-Resolved de Novo Assembly Using Phased Assembly Graphs with Hifiasm. Nat Methods. 2021; 18(2): $170-75$

PubMed Abstract | Publisher Full Text | Free Full Text

Chow W, Brugger K, Caccamo M, et al.: gEVAL - a Web-Based Browser for Evaluating Genome Assemblies. Bioinformatics. 2016; 32(16): 2508-10. PubMed Abstract | Publisher Full Text | Free Full Text

Cini A, Cappa F, Petrocelli I, et al.: Competition between the Native and the 
Introduced hornets Vespa crabro and Vespa Velutina: A Comparison of Potentially Relevant Life-History Traits. Ecol Entomol. 2018; 43(3): 351-62. Publisher Full Text

Edwards R: Social Wasps: Their Biology and Control. Rentokil Ltd. 1980. Reference Source

Foster KR, Seppä P, Ratnieks FLW, et al.: Low paternity in the hornet Vespa crabro indicates that multiple mating by queens is derived in vespine wasps. Behav Ecol Sociobiol. 1999; 46(4): 252-57.

Publisher Full Text

Foster KR, Gulliver J, Ratnieks FLW: Worker Policing in the European Hornet Vespa Crabro. Insect Soc. 2002; 49(1): 41-44.

Publisher Full Text

Garrison E, Marth G: Haplotype-Based Variant Detection from Short-Read Sequencing. arXiv:1207.3907. 2012.

Reference Source

Ghurye J, Rhie A, Walenz BP, et al.: Integrating Hi-C Links with Assembly Graphs for Chromosome-Scale Assembly. PLoS Comput Biol. 2019; 15(8): e1007273.

PubMed Abstract | Publisher Full Text | Free Full Text

Guan D, McCarthy SA, Wood J, et al.: Identifying and Removing Haplotypic Duplication in Primary Genome Assemblies. Bioinformatics. 2020; 36(9): 2896-98.

PubMed Abstract | Publisher Full Text | Free Full Text

Hoshiba H, Matsuura M, Imai HT: Karyotype Evolution in the Social Wasps Hymenoptera, Vespidae. JPN J Genet. 1989; 64(3): 209-22.

Publisher Full Text

Howe K, Chow W, Collins J, et al.: Significantly Improving the Quality of Genome Assemblies through Curation. GigaScience. 2021; 10(1): giaa153.

PubMed Abstract | Publisher Full Text | Free Full Text
Kerpedjiev P, Abdennur N, Lekschas F, et al.: HiGlass: Web-Based Visual Exploration and Analysis of Genome Interaction Maps. Genome Biol. 2018;

19(1): 125.

PubMed Abstract | Publisher Full Text | Free Full Text

Pawlyszyn B: Nest Relocation in the British Hornet Vespa Crabro Gribodoi Bequaert (Hym., Vespidae). Entomologist's Monthly Magazine. 1992.

Reference Source

Pawlyszyn B: Observations on the Life Cycle and Behaviour of the Hornet Vespa Crabro L. (Hym., Vespidae) in Gloucestershire. Entomologist's Monthly Magazine. 1994; 130: 159-63.

Reference Source

Rao SSP, Huntley MH, Durand NC, et al.: A 3D Map of the Human Genome at Kilobase Resolution Reveals Principles of Chromatin Looping. Cell. 2014 159(7): 1665-80

PubMed Abstract | Publisher Full Text | Free Full Text

Simão FA, Waterhouse RM, Ioannidis P, et al:: BUSCO: Assessing Genome

Assembly and Annotation Completeness with Single-Copy Orthologs.

Bioinformatics. 2015; 31(19): 3210-12.

PubMed Abstract | Publisher Full Text

Spredbery JP: Wasps: An Account of the Biology and Natural History of

Solitary and Social Wasps with Particular Reference to Those of the British Isles. Sidgwick \& Jackson. London. 1973.

Reference Source

Uliano-Silva M, Nunes JGF, Krasheninnikova K, et al.: marcelauliano/MitoHiFi: mitohifi v2.0. 2021

Publisher Full Text

Yoon KA, Kim K, Nguyen P, et al: Comparative Functional Venomics of Social Hornets Vespa Crabro and Vespa Analis. J Asia-Pac Entomol. 2015; 18(4):

815-23.

Publisher Full Text 


\section{Open Peer Review}

\section{Current Peer Review Status:}

\section{Version 1}

Reviewer Report 18 February 2022

https://doi.org/10.21956/wellcomeopenres.19402.r48317

(C) 2022 Nedoluzhko A. This is an open access peer review report distributed under the terms of the Creative Commons Attribution License, which permits unrestricted use, distribution, and reproduction in any medium, provided the original work is properly cited.

\section{Artem Nedoluzhko}

Russian Academy of Sciences, Saint Petersburg, Russian Federation

I thank the Wellcome Open Research authorities for handing me an opportunity to review a genome report: "The genome sequence of the European hornet, Vespa crabro Linnaeus, 1758". The report of high-level assembly of European hornet genome is clearly presented and I have only minor comments to the authors:

1. You should use genus Vespa name only one time per manuscript text then use abbreviation $V$. velutina and V. crabro.

2. More detailed information about the invasion of V. velutina to Europe should be added (e.g. PMID: 33177635). ${ }^{1}$

3. Explain why did you use a female hornet (a few words about sex determination in Hymenoptera)?

4. The place of specimen collection is presented twice in the report.

5. Please change citation for BUSCO v.5. ${ }^{2}$

\section{References}

1. Jones $E$, Conyers $C$, Tomkies $V$, Semmence $N$, et al.: Managing incursions of Vespa velutina nigrithorax in the UK: an emerging threat to apiculture. Scientific Reports. 2020; 10 (1). Publisher Full Text

2. Manni M, Berkeley MR, Seppey M, Simão FA, et al.: BUSCO Update: Novel and Streamlined Workflows along with Broader and Deeper Phylogenetic Coverage for Scoring of Eukaryotic, Prokaryotic, and Viral Genomes.Mol Biol Evol. 2021; 38 (10): 4647-4654 PubMed Abstract I Publisher Full Text

Is the rationale for creating the dataset(s) clearly described? 
Yes

Are the protocols appropriate and is the work technically sound?

Yes

Are sufficient details of methods and materials provided to allow replication by others? Yes

Are the datasets clearly presented in a useable and accessible format?

Yes

Competing Interests: No competing interests were disclosed.

Reviewer Expertise: evolutionary genomics, paleogenomics

I confirm that I have read this submission and believe that I have an appropriate level of expertise to confirm that it is of an acceptable scientific standard. 